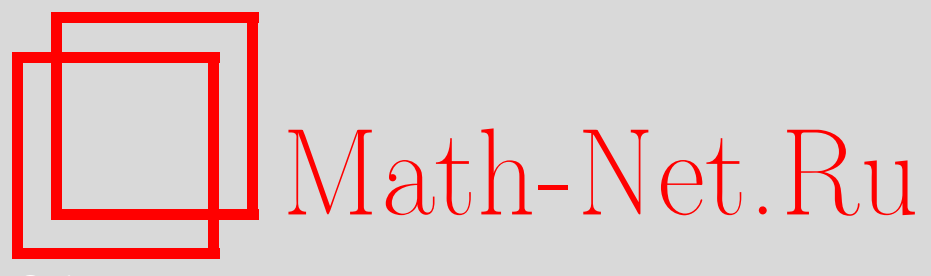

С. М. Гусейн-Заде, В. Эбелинг, Фильтрация, определяемая дугами на пространстве, УМН, 2006, том 61, выпуск 2, 163-164

DOI: https://doi.org/10.4213/rm1732

Использование Общероссийского математического портала Math-Net.Ru подразумевает, что вы прочитали и согласны с пользовательским соглашением http://www . mathnet.ru/rus/agreement

Параметры загрузки:

IP : 54.224 .187 .69

26 апреля 2023 г., 13:45:46

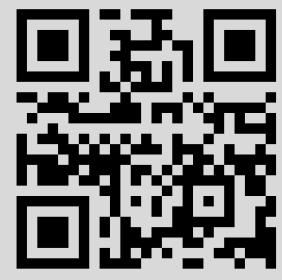




\section{Фильтрация, определяемая дугами на пространстве}

\section{С. М. Гусейн-Заде, В. Эбелинг}

Пусть $(V, 0)$ - росток комплексно-аналитического пространства. На кольце $\mathscr{O}_{V, 0}$ ростков функций на нем имеется фильтрация степенями максимального идеала. Мы определяем другую естественную фильтрацию, связанную с геометрией дуг на ростке $(V, 0)$, и описываем некоторые ее свойства.

Дуга $\phi$ на ростке $(V, 0)$ - это росток аналитического отображения $\phi:(\mathbb{C}, 0) \rightarrow(V, 0)$. Порядок $v_{\phi}(g)$ функции $g \in \mathscr{O}_{V, 0}$ на дуге $\phi$ определен как порядок суперпозиции $g \circ \phi$, т.е. как степень первого нетривиального слагаемого в степенном разложении $g \circ \phi(\tau)=$ $a \tau^{v_{\phi}(g)}+\cdots, a \neq 0$ (если $g \circ \phi \equiv 0$, то $\left.v_{\phi}(g):=+\infty\right)$. Пусть $\widehat{v}(g):=\min _{\{\phi\}} v_{\phi}(g)$. Для ростка $g \neq 0$ число $\widehat{v}(g)$ всегда конечно.

ОПРедЕлЕниЕ. Дуговая фильтрачия (arc filtration по английски) $\mathscr{O}_{V, 0}=F_{0} \supset$ $F_{1} \supset F_{2} \supset \cdots$ на кольце $\mathscr{O}_{V, 0}$ ростков функций - это фильтрация идеалами $F_{i}=\{g \in$ $\left.\mathscr{O}_{V, 0} \mid \widehat{v}(g) \geqslant i\right\}$.

ЗАмечАниЕ. Определенная аналогичным образом фильтрация имеется также на модуле ростков 1-форм на пространстве.

Пусть росток $(V, 0)$ имеет изолированную особую точку в нуле, и пусть $\pi:(X, D) \rightarrow$ $(V, 0)$ - разрешение особенности, где $D=\pi^{-1}(0)=\bigcup_{i=1}^{r} E_{i}$ - дивизор с нормальными пересечениями, $E_{i}(i=1, \ldots, r)$ - его неприводимые компоненты. Пусть $v_{i}(g)(g \in$ $\left.\mathscr{O}_{V, 0}\right)$ - порядок поднятия $\widetilde{g}=g$ 。 $\pi$ функции $g$ вдоль компоненты $E_{i}$ исключительного дивизора. Тем самым на кольце $\mathscr{O}_{V, 0}$ ростков функций определены семейство нормирований $\left\{v_{1}, \ldots, v_{r}\right\}$ и мульти-индексная фильтрация: $J(\underline{v})=\left\{g \in \mathscr{O}_{V, 0} \mid \underline{v}(g) \geqslant \underline{v}\right\}$, $\underline{v}=\left(v_{1}, \ldots, v_{r}\right), \underline{v}(g)=\left(v_{1}(g), \ldots, v_{r}(g)\right)$ (см., например, [1]).

\section{ПрЕДЛОЖЕНИЕ 1.}

$$
\widehat{v}(g)=\min _{1 \leqslant i \leqslant r} v_{i}(g) .
$$

ДоказАтельство. Это следует из того, что множество дуг на ростке $(V, 0)$ совпадает с множеством дуг на $(X, D)$, а порядок функции $g$ вдоль компоненты $E_{i}$ равен ее порядку вдоль гладкой кривой общего положения, трансверсальной к $E_{i}$.

Пусть

$$
P_{V, 0}(t)=\sum_{i=0}^{\infty} \operatorname{dim}\left(F_{i} / F_{i+1}\right) \cdot t^{i}
$$

- ряд Пуанкаре дуговой фильтрации на кольце $\mathscr{O}_{V, 0}$.

ЗАмечАниЕ. Коэффициент $\operatorname{dim}\left(F_{1} / F_{2}\right)$ при $t$ равен размерности подпространства касательного пространства $T_{0} V$, порожденного касательными векторами $\dot{\phi}(0)$ гладких дуг $\phi:(\mathbb{C}, 0) \rightarrow(V, 0)$.

В [2], [1] был определен (обобщенный) ряд Пуанкаре $P_{\left\{v_{i}\right\}}\left(t_{1}, \ldots, t_{r}\right)$, соответствующий набору нормирований $\left\{v_{i}\right\}$.

Редукиией $\bar{P}(t)$ формального степенного ряда от нескольких переменных $P\left(t_{1}, \ldots\right.$, $\left.t_{r}\right)\left(\underline{t}=\left(t_{1}, \ldots, t_{r}\right)\right)$ назовем степенной ряд одной переменной $t$, получаемый из ряда $P\left(t_{1}, \ldots, t_{r}\right)$ заменой каждого монома $\underline{t}^{\underline{v}}=t_{1}^{v_{1}} \cdots t_{r}^{v_{r}}$ в нем мономом $t^{\min v_{i}}$. Например, редукцией ряда $P\left(t_{1}, t_{2}\right)=1+t_{1} t_{2}^{2}+t_{1}^{2} t_{2}$ является $\bar{P}(t)=1+2 t$.

ПреДЛОЖенИЕ 2. Пустъ росток $(V, 0)$ неприводим. Тогда ряд Пуанкаре $P_{V, 0}(t)$ дуговой фильтрации совпадает с редукцией $\overline{P_{\left\{v_{i}\right\}}}(t)$ ряда Пуанкаре $P_{\left\{v_{i}\right\}}\left(t_{1}, \ldots, t_{r}\right)$ набора $\left\{v_{i}\right\}$ дивизорных нормирований.

Первый автор поддержан DFG-программой "Глобальные методы в комплексной геометрии" (Еb 102/4-2), грантами РФФИ-04-01-00762 и НШ-1972.2003.1. 
ДокАЗАтЕльство. Выразим оба эти ряда Пуанкаре в виде интегралов по отношению к эйлеровой характеристике по проективизации $\mathbb{P} \mathscr{O}_{V, 0}$ пространства $\mathscr{O}_{V, 0}$ (см., например, [1]). Из [1] следует, что

$$
P_{\left\{v_{i}\right\}}\left(t_{1}, \ldots, t_{r}\right)=\int_{\mathbb{P} \mathscr{O}_{V, 0}} \underline{t}^{\underline{v}(g)} d \chi, \quad P_{V, 0}(t)=\int_{\mathbb{P} \mathscr{O}_{V, 0}} t^{\widehat{v}(g)} d \chi .
$$

Предложение 2 следует из формул (1) и предложения 1.

Утверждение предложения 2, вообще говоря, не имеет места для приводимых ростков пространств. Причина состоит в том, что для делителя нуля $g$ моном $\underline{t}^{-(g)}$ в первом из интегралов (1) должен считаться равным нулю, в то время как $t^{\widehat{v}(g)}$ во втором отлично от нуля.

СледствиЕ. Редукиия $\overline{P_{\left\{v_{i}\right\}}}(t)$ ряда Пуанкаре $P_{\left\{v_{i}\right\}}\left(t_{1}, \ldots, t_{r}\right)$ набора дивизорных нормирований $\left\{v_{i}\right\}$ не зависит от разрешения особенности.

Для рациональных особенностей поверхностей ряды Пуанкаре $P_{\left\{v_{i}\right\}}\left(t_{1}, \ldots, t_{r}\right)$ были вычислены в [1]. В применении к ним предложение 2 дает следующее утверждение.

ПредложениЕ 3. Для рациональных двойных точек поверхностей ряды Пуанкаре $P_{V, 0}(t)$ дуговой фильтрачии даются следующей таблищей: $A_{k}: \frac{1-t^{2}}{(1-t)^{3}}, D_{k}$ : $\frac{1-t^{k-1}}{(1-t)^{2}\left(1-t^{k-2}\right)}, \quad E_{6}: \frac{1-t^{4}}{(1-t)\left(1-t^{2}\right)^{2}}, \quad E_{7}: \frac{1-t^{6}}{(1-t)\left(1-t^{2}\right)\left(1-t^{4}\right)}, \quad E_{8}: \frac{1-t^{6}}{\left(1-t^{2}\right)^{2}\left(1-t^{3}\right)}$.

ЗАмечАния. 1. В процессе вычислений на каждом шаге получаются выражения, не являющиеся произведениями/отношениями циклотомических многочленов. Однако в конце они приводят к рядам, являющимся такими произведениями.

2. Ряд Пуанкаре $P_{V, 0}(t)$ дуговой фильтрации не обязан быть произведением/отношением циклотомических многочленов. Например, для мономиальной пространственной кривой, заданной как образ отображения $x \mapsto\left(x^{3}, x^{4}, x^{5}\right)$, имеем $P_{V, 0}(t)=$ $\left(1-t+t^{3}\right) /(1-t)$. Однако для неприводимой особенности плоской кривой ряд Пуанкаре $P_{V, 0}(t)$ является рядом указанного вида.

3. Пусть $(V, 0)$ - неприводимая особенность с хорошим $\mathbb{C}^{*}$-действием (т.е. 0 принадлежит замыканию каждой орбиты). Если $\mathbb{C}^{*}$-действие свободно вне начала координат, дуговая фильтрация совпадает с фильтрацией, определенной "квазиоднородной" градуировкой на кольце $\mathscr{O}_{V, 0}$, соответствующей $\mathbb{C}^{*}$-действию. Если действие не свободно, это, вообще говоря, не так.

4. Для всех рациональных двойных точек ряд Пуанкаре $P_{V, 0}(t)$ (как рациональная функция от $t$ ) имеет степень -1 и полюс второго порядка в точке $t=1$. При этом эти ряды совпадают с рядами Пуанкаре квазиоднородной градуировки для других рациональных двойных точек. Это соответствие выглядит следующим образом:

$$
A_{k} \mapsto A_{1}, \quad D_{k} \mapsto A_{k-2}, \quad E_{6} \mapsto A_{3}, \quad E_{7} \mapsto A_{5}, \quad E_{8} \mapsto D_{4}
$$

Смысл этого соответствия непонятен.

В работе [3] ряды Пуанкаре дуговой фильтрации были вычислены для особенностей поверхностей из списков В.И. Арнольда, включая все уни- и бимодальные. Классификация унимодальных особенностей по рядам Пуанкаре оказалась согласованной с иерархией Э. Брискорна, основанной на примыканиях особенностей.

\section{Список литературы}

[1] A. Campillo, F. Delgado, S. M. Gusein-Zade, Invent. Math., 155:1 (2004), 41-53. [2] A. Campillo, F. Delgado, K. Kiyek, Manuscripta Math., 83:3-4 (1994), 405-423. [3] W. Ebeling, S. M. Gusein-Zade, Math. Proc. Cambridge Philos. Soc., 138:2 (2005), 307-314.

\section{C. М. Гусейн-Заде (S. M. Gusein-Zade)}

Московский государственный университет им. М. В. Ломоносова

E-mail: sabir@mccme.ru

В. Эбелинг (W. Ebeling)

Universität Hannover, Institut für Mathematik

E-mail: ebeling@math.uni-hannover.de
Представлено В. М. Бухштабером Принято редколлегией 01.02 .2006 